\title{
RESEARCH
}

Open Access

\section{Routine pre-employment echocardiography assessment in young adults: cost and benefits}

\author{
Ahmed Gaafar ${ }^{1 *}$ (D) and Asmaa Gaafar ${ }^{2}$
}

\begin{abstract}
Background: Conventional echocardiography is a safe, available, and accurate tool for cardiac structural and functional evaluation, but it should not cancel clinical assessment and history tacking, and indeed both are complementary. A pre-employment assessment is important for employees and community safety and suitability for a specific work requirement.
\end{abstract}

Results: Aiming to assess the value of routine pre-employment echocardiography for the detection of cardiac abnormalities, we examined seven hundred ninety-five persons who were routinely referred to us for preemployment conventional echocardiography. Only 9 persons had structural cardiac abnormalities (1.3\%) and distributed as follows: two had bicuspid aortic valve with isolated aortic regurgitation, one of them had mild AR, and the other had moderate AR. Two cases had mitral valve prolapse, one of them had trivial MR, while the other had a flail anterior leaflet with severe MR. One patient had atrial septal defect $1.5 \mathrm{~cm}$ with mild pulmonary hypertension and right-sided chambers dilatation. One patient had dextrocardia (situs inversus totalis) without other cardiac problems. One had moderate pulmonary hypertension and modest right-sided chambers dilation. Two patients had left ventricular hypertrophy. Surprisingly, we did not detect rheumatic valvular heart disease. The money cost of echocardiography tests for those 795 persons was 198,750 Egyptian pounds (LE); their transportation cost was about 19.800LE. The total group time cost of the tests was $265 \mathrm{~h}$, total time lost at the waiting room was $1590 \mathrm{~h}$, total transportation time lose was $2385 \mathrm{~h}$, so the total time cost was about $4000 \mathrm{~h}$. Using psychological stress questionnaire, 33 participants (4.2\%) had results suggestive of a low sense of psychological pressure due to echocardiography examination, 221 participants (27.8\%) had results suggestive of a moderate feeling of stress, while 541 participants (68\%) had results suggestive of a high sense of stress.

Conclusion: We recommend against routine echocardiography for cardiac assessment in pre-employment assessment and to do it only for persons with abnormal clinical or ECG findings.

Keywords: Echocardiography, Pre-employment assessment psychological stress, Money cost

\footnotetext{
* Correspondence: Gaafar911@hotmail.com

${ }^{1}$ Cardiology Department, Faculty of Medicine, Helwan University, Cairo, Egypt

Full list of author information is available at the end of the article
}

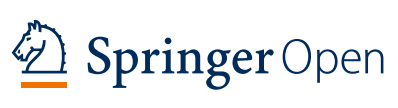

(c) The Author(s). 2021 Open Access This article is licensed under a Creative Commons Attribution 4.0 International License, which permits use, sharing, adaptation, distribution and reproduction in any medium or format, as long as you give appropriate credit to the original author(s) and the source, provide a link to the Creative Commons licence, and indicate if changes were made. The images or other third party material in this article are included in the article's Creative Commons licence, unless indicated otherwise in a credit line to the material. If material is not included in the article's Creative Commons licence and your intended use is not permitted by statutory regulation or exceeds the permitted use, you will need to obtain permission directly from the copyright holder. To view a copy of this licence, visit http://creativecommons.org/licenses/by/4.0/. 


\section{Background}

Assessment of fitness for work definition is the assessment of a worker's capacity to work without risk to their own or others' health and safety. The medical examination offers several purposes for the benefit of the employer and the employee. It is done to ensure that the prospective employee has no "serious medical condition" and he is "physically and mentally" suitable for the job offered. But in some jobs, the demands of the work requirements that the prospective employee should meet the minimum required medical standards to qualify for the appointment [1].

Despite the argument about the practical benefits of screening tests, there is a true desire to screen populations under cardiovascular risk; the screening aims to detect underlying diseases or abnormalities and subsequently reduce morbidity and mortality, but unfortunately, there is no agreement about the most proper and reliable test which can effectively detect the disease or abnormalities [2].

The leading cause of sudden cardiac death is well known which is a cardiovascular disease as silent ischemic heart disease, lethal arrhythmias, and myocardial diseases as hypertrophic cardiomyopathy, so early detection, diagnosis, and proper management of silent cardiovascular diseases are of great importance [3].

The AHA guidelines recommended a fourteen elements comprehensive medical questionnaire of the personal and family history and physical examination as a guide for examiners to use it when they perform the pre-participation examinations for adolescent athletes (Table 1) [4].

Also, the US military recommended mandatory screening for cardiovascular disease military recruits using screening questionnaires and physical examination, but ECG is only performed in selective cases guided by history, symptoms, or physical data, and as a routine for aviation duty and in soldiers $>40$ years old [2].

Nistri et al. used data from 34,910 persons $\geq 18$ years old at the military screening program from 1992 to 1996 based on history-taking, physical examination, and routine ECG, and they found $8 \%$ had abnormalities and were referred for echocardiographic examination; echocardiography revealed that $0.7 \%$ of them had a new HCM diagnosis [5].

Unfortunately, personal history, family history, and physical examination have low sensitivity to detect many diseases and abnormalities that can cause sudden cardiac death [6].

A retrospective study of suddenly died competitive athletes revealed only $<5 \%$ of them had a confirmed cardiovascular diagnosis after screening with standard history and physical examination [7].

For example, by ECG only you can detect ion channelopathies and Wolff Parkinson-White syndrome. Many structural cardiac diseases which may lead to SCD, symptoms,
Table 1 The 14-element AHA recommendation for preparticipation cardiovascular screening of competitive athletes Medical history (parental verification recommended for high school and middle school athletes)

\section{Personal 1.Exertional chest pain/discomfort history \\ 2.Exertional syncope or near-syncope \\ 3.Excessive exertional and unexplained fatigue/fatigue associated with exercise \\ 4.Prior recognition of a heart murmur \\ 5.Elevated systemic blood pressure \\ 6.Prior restriction from participation in sports \\ 7.Prior testing for the heart ordered by a physician \\ Family 8.Premature death-sudden and unexpected before age \\ history $\quad 50$ year due to heart disease, in one or more relatives \\ 9.Disability from heart disease in a close relative $<50$ years old \\ 10.Specific knowledge of certain cardiac conditions in family members: hypertrophic or dilated cardiomyopathy, long QT syndrome or other ion channelopathies, Marfan syndrome, or clinically important arrhythmias \\ Physical exam \\ 11. Heart Murmur-exam supine and standing or with Valsalva, specifically to identify murmurs of dynamic $L$ ventricular outflow tract obstruction \\ 12. Femoral pulses to exclude aortic stenosis \\ 13. Physical stigmata of Marfan syndrome \\ 14. Brachial artery blood pressure (sitting, preferably taken in both arms)}

Positive/abnormal screen warrants further evaluation and 12-lead EKG AHA does Not currently recommend routine 12-lead ECG

family history of heart disease, and clinical signs are infrequent findings. For example in HCM, a systolic murmur is audible at rest in $25 \%$ of patients and with dynamic auscultation, it is audible in an additional 50\% [8].

The British Army screening system although they described transthoracic echocardiography as relatively cheap, fast, and accessible in cardiac screening for potential recruits, they considered the need for professionals specialized in echocardiography increases the cost of the screening system and reduces the number of patients that can be investigated. Their final recommendation was to perform routine echocardiography only when the cost is a less important factor and the numbers of participants are low, as screening professional football players [9]. So, the International Federation of Association Football (FIFA), the International Cycling Union (UCI), and the U.S. National Basketball Association (NBA) perform routine echocardiography in the primary screening tests in asymptomatic athletes [10].

The pre-employment screening test is just one factor of many factors used to judge the suitability of someone for the job. We cannot ignore the psychological stress induced by the pre-employment test. "Fear of failure to 
individuals who tie their self-worth to the outcome of pre-employment assessment" is particularly true for prospective employees, as the evaluative and competitive nature of the process of job application often causes feelings of anxiety, frustration, and distress [11].

\section{Aim of the study}

We aimed to assess the usefulness and cost of using echocardiography as routine practice in pre-employment assessment.

\section{Methods}

This a prospective survey study included 795 young persons (772 males, 23 females), with an age range between 20 and 31 years referred for routine echocardiography assessment as a screening test before employment in a variety of manual work jobs on railway lines and maintenance workshops of Egypt Railways, at the period between $1 / 2020$ and $4 / 2020$.

All persons were subjected to full history tacking using the AHA questionnaire for the cardiovascular screening of young athletes before competitive sports (Table 1) [12].

Also, a full cardiac examination was done for all subjects with special concerns for BP, cardiac murmurs, and dynamic auscultation to avoid missing LVOT obstruction, also a routine assessment of peripheral and femoral pulsations to detect aortic coarctation.

Routine 12-lead ECG was obtained to assess electrical abnormalities, myocardial ischemia, or chambers abnormalities. We did ECG at speed $25 \mathrm{~mm} / \mathrm{s}$ with normal standardization $(10 \mathrm{~mm} / 1 \mathrm{mV})$ using automated mode.

Using the recommendations of last published American Society of Echocardiography [13], conventional transthoracic two-dimensional echocardiography using Philips HD11 (Philips Medical Systems; Eindhoven, Netherland) machine was done routinely; we used basic echocardiographic views (left parasternal long and short axis, apical 4, apical 2, and apical 3 chambers views, subcostal and suprasternal views) while the subjects at a left lateral position and ECG gated to assess chambers sizes, myocardial mass, and LV wall thickness, ventricular systolic function (by MMode, and area length method when needed) and diastolic function (using pulsed-wave Doppler and pulsed wave tissue Doppler to calculate E/A, E'/A' and E/E'), valvular morphologies and functions, great vessels diameters, pericardium, and finally to exclude congenital abnormalities.

The echocardiography test cost and transportation cost of each person were obtained to be used for the calculation of total cost.

The time of echocardiography exam, transportation time, and waiting time for each subject was recorded.

As regards our knowledge, we did not find a questionnaire focusing on psychological stress induced by peemployment medical assessment, so we developed the Pre-employment psychological Stress Scale (PSS) to assess and escalating the psychological stress induced by pre-employment medical assessment (Table 2).

The questionnaire was used to assess the person's psychological stress due to referral for echocardiography

Table 2 Psychological stress questionnaire

\begin{tabular}{|c|c|c|c|c|}
\hline & Item & Agree & Rather agree & Disagree \\
\hline 1 & I feel confident in my efficiency. & & & \\
\hline 2 & Sometimes I worry about my health. & & & \\
\hline 3 & My thinking about the consequences of a medical examination makes me nervous. & & & \\
\hline 4 & I see that the administrative decision to accept the job is unfair. & & & \\
\hline 5 & I am afraid not to secure an adequate physical level if I am not employed. & & & \\
\hline 6 & I have negative thoughts such as expectation of rejection or illness & & & \\
\hline 7 & I am scared of refusing to hire me. & & & \\
\hline 8 & I'm afraid my situation will get worse if I don't get the job. & & & \\
\hline 9 & I feel that the medical examination of the job is annoying and disturbing. & & & \\
\hline 10 & I am afraid of something that threatens my job. & & & \\
\hline 11 & I am afraid that the result of the medical examination will affect my private life. & & & \\
\hline 12 & I am concerned to think that I am seriously ill. & & & \\
\hline 13 & I fear my relationship with my family will get worse if I don't get a job. & & & \\
\hline 14 & I see the medical examination as a threat to some. & & & \\
\hline 15 & I am afraid not to get another job if I am refused. & & & \\
\hline
\end{tabular}

The total score of the questionnaire ranges between (15) minimum score, and (45) degree maximum, where it indicates:

- The range of degrees (15-25) suggested a low level of psychological stress

- The range of degrees (26-36) suggested a medium level of psychological stress

- The range of degrees (37-45) suggested a high level of psychological stress 
assessment. It contains (15) items, and suggested three alternatives for the answer (agree, rather agree, disagree), Key correction $(3,2,1)$.

* The total score of the questionnaire ranges between 15 and $45^{\circ}$, where it indicates:

- The range of degrees (15-25) suggested a low level of psychological stress.

- The range of degrees (26-36) suggested a medium level of psychological stress.

- The range of degrees (37-45) suggested a high level of psychological stress.

An exploratory study was conducted on an experimental sample representing the original study sample for the following aims:

Determining the psychometric properties of the scale used in the study, so the authors can carry out the basic study through a scale of enough validity and stability.

The exploratory sample represents the individuals of the basic sample in all its specifications (gender and age distributions) to standardize study scale on them through validity and stability inappropriate ways. The exploratory sample consisted of (80) individuals, males and females (78 males $79.5 \%$ and 2 females $2.5 \%$ ), whose ages ranged in the age group (24-33) years so that the individuals of the basic sample were represented in all their characteristics.

\section{Statistical methodology}

Data were collected, revised, coded, and entered into the statistical package for social science (SPSS) version 21. Qualitative (categorical) data were presented as numbers and percentages; quantitative (continuous) data were presented as mean and standard deviations. The $p$ values were considered significant if less than 0.05 .

The test of the internal consistency of the questionnaire revealed that all 15 items are highly correlated with the total score. The Cronbach's alpha for the inventory was (0.77) indicating a respectable degree of internal consistency. The alpha for the males was (0.78), and for females was (0.75). A re-test after 6 weeks showed a significant correlation $(r=$ $0.64, p>0.01$ ) between test and re-test scores. This allows the authors to use the scale in the current study.

\section{Results}

Aiming to assess the value of routine pre-employment echocardiography for the detection of cardiac abnormalities, we examined seven hundred ninety-five young persons who were routinely referred to us for pre-employment conventional echocardiography.

Mean heart rate of the studied subjects was $85.2 \pm 9.8$, (range, 63-120) and categorized them into three groups, group 1: HR of $63-80 \mathrm{~b} / \mathrm{m}$ and included 233 subjects (29.3\%); group 2: HR of $81-100$ and included 417 subjects (52.5\%); and group 3: HR of $101-120 \mathrm{~b} / \mathrm{m}$ and included 145 subjects (18.2\%).

Seven hundred seventy-four persons (97.4\%) were male and $21(2.6 \%)$ were female with a mean age was 25 \pm 8.4 years old. Further, 193 (24\%) persons were smokers; all of them were men. Seven $(0.9 \%)$ persons had elevated BP at the examination; two of them were known to be hypertensive patients on medical treatment and the other five subjects had no history of hypertension and were referred to a hypertension clinic to complete their assessment. Two persons $(0.25 \%)$ were diabetics; one was newly discovered and the other one was known type $1 \mathrm{DM}$ on medical treatment (Table 3).

By history, four $(0.5 \%)$ subjects had a family history of SCD; only two of them occurred at a young age (undiagnosed) while the other two SCD cases occurred at age 65 and 69 (due to ACS); the two patients with a family history of SCD at a young age had normal ECG cardiac examination and normal echocardiography without a history of any syncopal attacks. Three $(0.37 \%)$ subjects had a family history of premature IHD. Two $(0.25 \%)$ persons had a history of single attack of syncope were diagnosed as neurogenic syncope (one of them syncopal attack occurred during hard work in hot weather and the other during prolonged fasting when he was in longstanding in a queue).

ECG assessment revealed 14 subjects (1.7\%) had abnormal ECGs, three subjects had infrequent PVCs, two subjects had LVH, one subject had RBBB, one subject had dextrocardia pattern, two subjects had abnormal cardiac axis, four persons had non-specific $\mathrm{T}$ wave changes, and one subject had RVH (Table 4).

Table 3 Baseline demographic data of the whole study population

\begin{tabular}{ll}
\hline Demographic data & All patients \\
\hline Count (\%) & $795(100 \%)$ \\
Age (years) & $25 \pm 8.4$ \\
Mean \pm SD & $24-33$ \\
Range & \\
Gender & $774(97.4 \%)$ \\
Male & $21(2.6 \%)$ \\
Female & \\
Risk factors and history & $7(0.9 \%)$ \\
Elevated BP & $2(0.25 \%)$ \\
DM & $193(24 \%)$ \\
Smoking & $4(0.5 \%)$ \\
Family history of SCD & $3(0.37 \%)$ \\
Family history of premature IHD & $2(0.25 \%)$ \\
Syncope & \\
\hline
\end{tabular}

$B P$ blood pressure, $D M$ diabetes mellitus, $S C D$ sudden cardiac death, $I H D$ ischemic heart disease 
Table 4 Baseline clinical, ECG, echocardiographic, and laboratory data

\begin{tabular}{|c|c|}
\hline $\begin{array}{l}\text { Clinical, ECG, echocardiographic } \\
\text { and laboratory data }\end{array}$ & All patients \\
\hline \multicolumn{2}{|l|}{$\mathrm{SBP}(\mathrm{mmHg})$} \\
\hline Mean \pm SD & $107.1 \pm 18.0$ \\
\hline Range & $90-150$ \\
\hline \multicolumn{2}{|l|}{$\mathrm{DBP}(\mathrm{mmHg})$} \\
\hline Mean \pm SD & $87.5 \pm 12.4$ \\
\hline Range & $60-100$ \\
\hline \multicolumn{2}{|l|}{ Pulse (beat/min) } \\
\hline Mean \pm SD & $85.2 \pm 9.8$ \\
\hline Range & $63-120$ \\
\hline \multicolumn{2}{|l|}{ ECG } \\
\hline Normal & $781(98.3 \%)$ \\
\hline Abnormal & $14(1.7 \%)$ \\
\hline \multicolumn{2}{|l|}{ Echocardiography } \\
\hline EF (\%) mean \pm SD (range) & $62.9 \pm 7.8(54-76)$ \\
\hline LVEDd (cm) & $4.9 \pm 0.81$ \\
\hline IVSd $(\mathrm{cm})$ & $0.82 \pm 0.21$ \\
\hline PWd (cm) & $0.79 \pm 0.18$ \\
\hline LA area $\left(\mathrm{cm}^{2}\right)$ & $17 \pm 3.1$ \\
\hline $\mathrm{RVd}(\mathrm{cm})$ & $3.2 \pm 1.1$ \\
\hline TV max systolic velocity $(\mathrm{m} / \mathrm{s})$ & $2.1 \pm 4.7$ \\
\hline MV E/E' (range) & $6.2 \pm 3.1(3.2-8.9)$ \\
\hline \multicolumn{2}{|l|}{ Lab mean \pm SD } \\
\hline Creatinine (mg/dL) & $0.8 \pm 0.24$ \\
\hline $\mathrm{Hb}(\mathrm{gm} / \mathrm{dl})$ & $11 \pm 3.7$ \\
\hline TSH (IU/mL) & $2.7 \pm 1.8$ \\
\hline
\end{tabular}

SBP systolic blood pressure, $D B P$ diastolic blood pressure, EF ejection fraction, LVEDd left ventricular end diastolic diameter, IVSd interventricular septum thickness at diastole, Pwd posterior wall thickness at diastole, LA left atrium, $R V d$ right ventricular basal diameter at apical 4 chamber view, $T V$ tricuspid valve, $M V$ mitral vlve, E/E' mitral valve $E$ wave by pulsed wave Doppler/mitral valve $E^{\prime}$ wave by annular pulsed tissue Doppler, $H b$ hemoglobin, $T S H$ thyroid-stimulating hormone
Echocardiography assessment revealed only nine persons (1.3\%) with structural cardiac abnormalities, two had bicuspid aortic valve with isolated aortic regurgitation (AR), one of them had mild AR, and the other had moderate AR. Two cases of mitral valve prolapse (MVP); one of them had trivial MR while the other had flail anterior leaflet due to ruptured chordae with severe MR. One patient had atrial septal defect $1.5 \mathrm{~cm}$ with mild pulmonary hypertension (ESPAP $=40 \mathrm{mmHg}$ ) and right-sided chambers dilatation (RA area $=26 \mathrm{~cm}^{2}$, RV basal transverse diameter at apical four-chamber view = $4.7 \mathrm{~cm}$ ). One patient had dextrocardia (situs inversus totalis) without other cardiac abnormalities. One had pulmonary hypertension (ESPAP $=50 \mathrm{mmHg}$ ) and right-sided chambers dilation (RA area $=22 \mathrm{~cm}^{2}$, RV basal transverse diameter at apical 4 chamber view $=4.6$ $\mathrm{cm}$ ) without apparent underlying cardiac abnormalities. Two patients had LVH, both were hypertensive subjects.

We did not detect rheumatic valvular heart disease. Also, we did not find HCM cases.

When we revised persons with abnormal findings, six of them had clear clinical abnormal findings and/or abnormal ECG, as shown in Table 5.

The money cost of echocardiography for those 795 persons was 198,750 Egyptian pounds (LE); their transportation cost was about 19.800LE. The total group time cost of the echocardiographic tests was $265 \mathrm{~h}$, total time lost at echocardiography waiting room was $1590 \mathrm{~h}$, total transportation time lose was $238 \mathrm{~h}$, so total time cost was about $4000 \mathrm{~h}$.

Psychological stress was another cost that they had to suffer from; every one of them was in a different degree of stress and fear of losing the job if there was an abnormal finding in the test. By using the psychological stress questionnaire, 33 participants (4.2\%) had a range of scores between (15-25), which suggested a low sense of psychological stress due to echocardiographic examination, while 221 persons (27.8\%) had a range of scores between (26-36), which suggested a moderate feeling of

Table 5 Baseline ECG and clinical data of the nine subjects with their abnormal echocardiographic findings

\begin{tabular}{llll}
\hline Echocardiographic abnormalities & No. & ECG findings & Clinical \\
\hline ASD & 1 & RVH & Splitted fixed S2 \\
Dextrocardia & 1 & Dextrocardia pattern & $\begin{array}{l}\text { Lt sided liver, heart sounds and LV apex at Rt side } \\
\text { of the chest }\end{array}$ \\
Mild MVP, mild MR & 1 & Normal & Normal exam \\
Flail MV, severe MR & 1 & Non-specific & Muffled S1, systolic apical murmur, dyspnea NYHA II \\
BAV, Mild AR & 1 & Normal & Normal exam \\
BAV, moderate AR & 1 & Normal & AR murmur \\
LVH & 2 & LVH & Elevated BP \\
Pulmonary hypertension & 1 & Normal & Normal exam \\
\hline
\end{tabular}

$A S D$ intra-atrial septal defect, $M V P$ mitral valve prolapse, $M R$ mitral regurgitation, $B A V$ bicuspid aortic valve, $A R$ aortic regurgitation, $L V H$ left ventricular hypertrophy, $R V H$ right ventricular hypertrophy, $L t$ left, Rt right, NYHA New York Heart Association, BP blood pressure 
psychological stress due to the medical examination, and 541 subjects (68\%) had a range of scores obtained between (37-45), which suggested a high sense of psychological stress because of a medical examination (Fig. 1).

\section{Discussion}

As we noticed, the rising trend in many companies and even in governmental companies to use echocardiography as a routine assessment tool before employment for all subjects irrespective to medical history or clinical examination for fear of employing an apparently healthy person undiscovered cardiovascular disease which will lead to higher medical insurance and financial costs, beside the risk on the employee and his colleagues or general population. So we designed this study to judge this practice in the light of our country with its limited resources. Also, the previous studies studied different population age and was done in western countries with better health care system and lower incidence of rheumatic heart disease. And as we expect that the results will support our recommendations to the authorities to stop this unnecessary practice to save time and cost and to avoid unnecessary psychological stress.

In our study, the fourteen elements questionnaire only revealed 13 persons with abnormal findings and this can be attributed to the socioeconomic factor with the fear of losing the job which is so valuable for them so they tended to deny the symptoms and their personal and family history of cardiovascular diseases despite their previous orientation by physicians as regards the safety purpose of these data.

As regard time cost, the total time consumed was about 4000 h ( $\approx 166.7$ day) which reflected as a significant delay on the services offered by echocardiographic unite for regular work.

And the total money spent for the examinations was 218550 Egyptian pounds which is considered a large burden in a developing country with subjects of low economic level, and in comparison with the few data added by echocardiography in the study, it looks unpractical and costly as a routine examination tool for detection of cardiovascular abnormalities in subjects without symptoms, clinical, and/or ECG abnormalities.

The study suggested that the participants suffered from various degrees of psychological stress caused by referral for echocardiography where $68 \%$ of them had severe psychological stress while $32 \%$ had mild to moderate psychological stress, which should be put into consideration while recommending this test.

From the 795 subjects who were routinely examined by echocardiography as a pre-employment test, only 9 subjects had abnormal findings; 6 of them already had abnormal clinical and/or ECG findings, so only three subjects $(0.377 \%)$ had cardiac abnormalities that were detected only by echocardiography but their abnormalities were non-serious or non-significant forms (Table 5).

Lindekleiv $\mathrm{H}$ et al.'s study found that echocardiographic screening the general population for structural heart disease had no benefit for death or the risk of myocardial infarction and CV stroke. The prevalence of structural heart disease was $7.6 \%$. They had a higher prevalence than our study due to the higher age of their studied group (mean age was 60 years) and their study contained a larger sample volume of 3272 subjects [14].

We found that $18 \%$ of the subjects had a heart rate be between 100 and $120 \mathrm{~b} / \mathrm{m}$ (sinus tachycardia), which

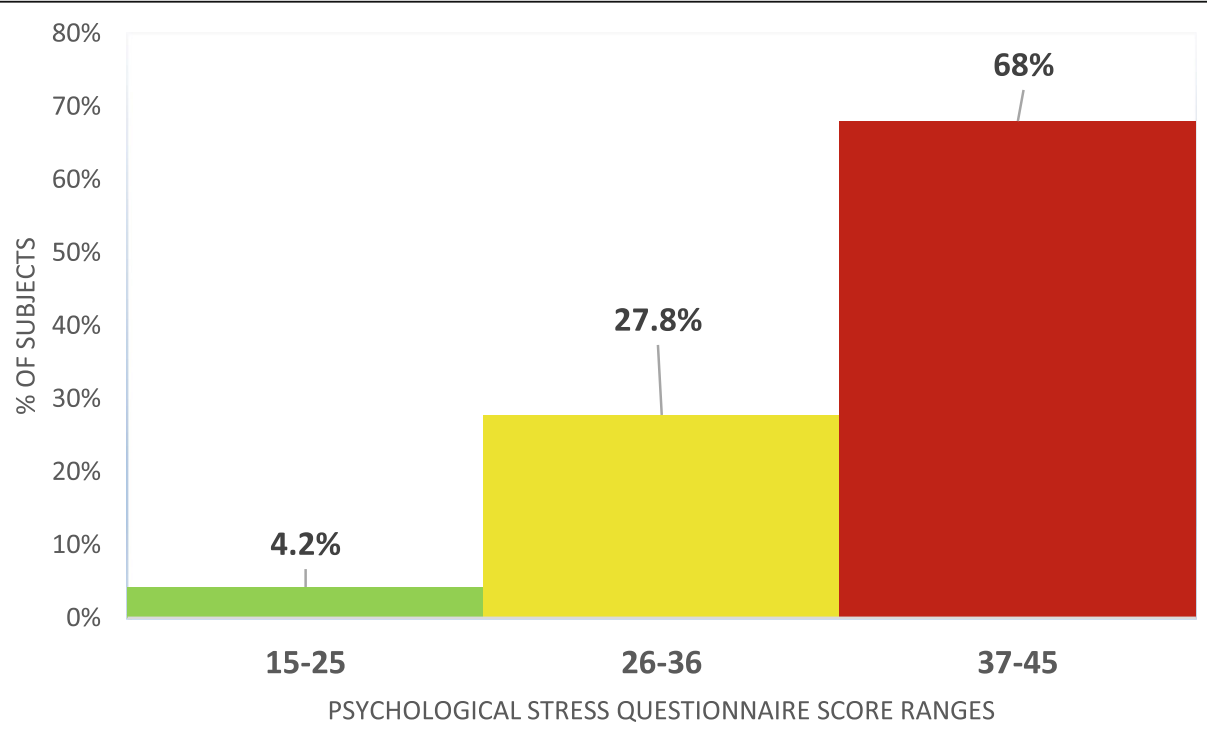

Fig. 1 Distribution of studied subjects as regard psychological stress questionnaire score range 
may reflect the increased psychological burden for fear of the rejection after the echocardiographic examination.

Of the seven subjects with elevated BP during screening, two were known to be hypertensive patients on medical treatment but not well controlled with evidence of LVH by ECG, and echocardiography confirmed the diagnosis of LVH in these two patients. Two of the remaining five subjects truly have grade 1 hypertension and were managed with lifestyle modifications, and their ECG and echocardiography were normal. The remaining 3 subjects had stress-induced elevated BP with normal home BP readings. In this group, echocardiography did not add new data.

Subjects with DM, family history of SCD, and family history of premature CAD were asymptomatic with normal cardiac examinations, ECGs, and echocardiography.

The two subjects with a history of single attack of syncope were diagnosed as neurogenic syncope (one of them syncopal attack occurred during hard work in hot weather and the other during prolonged fasting when he was in long-standing in a queue) without any other clinical, ECG or echocardiographic abnormalities.

As regards the 14 subjects with abnormal ECGs, five of them had abnormal echocardiographic findings (Table 4) while the others had normal echocardiography.

Despite the majority of examined subjects were from rural areas, we did not find rheumatic heart disease cases which were unexpected to the authors, but we tried to explain this by the wide empirical use of long-acting penicillin injection at a low threshold in children with symptoms mimic rheumatic fever or even just elevation in ESR or ASOT in the last few decades, but this number of subjects couldn't be enough to give such result, we think this point needs further screening with a much higher number of participants.

Also, hypertrophic cardiomyopathy (HCM) is the most common inherited heart disease; we did not find HCM cases and we attribute this to the relatively small study shamble for such disease with prevalence rates between 0.2 and $0.3 \%$.

Data coming from the 25-year Italian experience for screening to detect $\mathrm{HCM}$ in young competitive athletes stated that although echocardiography is the main diagnostic test for HCM diagnosis, it is expensive and impractical as a screening test for large populations [2] and they recommended twelve-lead ECG (in addition to history and physical examination) as an alternative, cost-effective method for population-based screening and stated that 12-lead ECG may be as sensitive as echocardiography in detecting $\mathrm{HCM}$ in the young athletic population. They put in their consideration the significant socio-economic impact and need for the cultural background [15].

Because of the low prevalence of structural heart disease among the general population, echocardiography has traditionally not been considered justified in low-risk individuals, although it is recommended in screening asymptomatic individuals with a family history of sudden death or hereditary diseases affecting the heart or the great vessels [16].

\section{Limitations}

Unequal gender distribution, limited age group, intraobserver variability, and the pretest bias of the examiners that young apparently healthy subjects will have normal examinations.

\section{Conclusion}

The use of echocardiography as a routine preemployment test added few data in patients without significant symptoms, ECG, and/or clinical findings, and on the other hand, it had significant economic and psychologic stress burden with unnecessary time consumption so we recommend against routine echocardiography for cardiac assessment in pre-employment assessment and to do it only for persons with symptoms suggestive of cardiac problems, abnormal clinical or ECG findings.

\section{Abbreviations}

BP: Blood pressure; DM: Diabetes mellitus; SCD: Sudden cardiac death; IHD: Ischemic heart disease; SBP: Systolic blood pressure; DBP: Diastolic blood pressure; EF: Ejection fraction; LVEDd: Left ventricular end-diastolic diameter; IVSd: Interventricular septum thickness at diastole; Pwd: Posterior wall thickness at diastole; LA: Left atrium; RVd: Right ventricular diameter by mode; TV: Tricuspid valve; MV: Mitral valve; E/E': Mitral valve E wave by pulsed-wave Doppler/mitral valve E' wave by annular pulsed tissue Doppler; Hb: Hemoglobin; TSH: Thyroid-stimulating hormone; ASD: Intra-atrial septal defect; MVP: Mitral valve prolapse; MR: Mitral regurgitation; BAV: Bicuspid aortic valve; AR: Aortic regurgitation; LVH: Left ventricular hypertrophy; RVH: Right ventricular hypertrophy; Lt: Left; Rt: Right; NYHA: New York Heart Association; RA: Right atrium; RV: Right ventricle; ESPAP: Estimated systolic pulmonary artery pressure; RBBB: Right bundle branch block; LVOT: Left ventricular outflow tract; HCM: Hypertrophic cardiomyopathy

\section{Acknowledgements}

Not applicable.

\section{Authors' contributions}

AhG performed the cardiac examination, history tacking, ECG interpretation, echocardiography assessment, and interpretation. AsG designed and interpreted the stress questionnaire. AhG and AsGa performed the study design and writing the manuscript and statistical analysis and interpretation. All authors read and approved the final manuscript.

\section{Funding}

None.

\section{Availability of data and materials}

The datasets used and/or analyzed during the current study are available from the corresponding author on reasonable request.

\section{Ethics approval and consent to participate}

The current study was conducted following Good Clinical Practice guidelines laid down in the Helsinki Declaration 1975 and Approval of Faculty of Medicine, Helwan University ethical committee (REC-FMHU) were obtained Verbal consent was obtained from the enrolled subjects (66-2020). The ethical committee when received the study protocol for approval, a clear statement that the subjects consents will be verbal consents was mentioned 
in the protocol and the investigator application form. The reason for using the verbal consents was the expected large number of study subjects.

\section{Consent for publication}

Not applicable.

\section{Competing interests}

The authors declare that they have no competing interests.

\section{Author details}

${ }^{1}$ Cardiology Department, Faculty of Medicine, Helwan University, Cairo, Egypt. ${ }^{2}$ Psychology Department, Faculty of Humanities, Al-Azhar University, Cairo, Egypt.

Received: 7 August 2020 Accepted: 26 December 2020

Published online: 06 January 2021

\section{References}

1. Cox RAF, Edwards FC, Palmer K (2009) Fitness for work: the medical aspects, 3rd edn. Oxford Medical Publications, New York

2. Levine BD, Okin PM, Saul JP, Van HGF, Mitten MJ (2014) Assessment of the 12-Lead electrocardiogram as a screening test for detection of cardiovascular disease in healthy general populations of young people (12 - 25 years of age), pp 1-36. https://doi.org/10.1016/j.jacc.2014.05.006

3. Maron BJ, Co-chair C, Zipes DP, Co-chair C (2005) 36th Bethesda conference: eligibility recommendations for competitive athletes with cardiovascular abnormalities. J Am Coll Cardiol 45:8. https://doi.org/10. 1016/j.jacc.2005.02.002

4. Madsen NL, Drezner JA, Salerno JC (2013) Sudden cardiac death screening in adolescent athletes: an evaluation of compliance with national guidelines. Br J Sports Med 47(3):172-177. https://doi.org/10.1136/bjsports2012-091670

5. Nistri S, Thiene G, Basso C, Corrado D, Vitolo A, Maron BJ (2003) Screening for hypertrophic cardiomyopathy in a young male military population. Am J Cardiol 91 (8):1021. https://doi.org/10.1016/S0002-9149(03)00132-2

6. Drezner JA, Fudge J, Harmon KG, Berger S, Campbell RM, Vetter VL (2012) Warning symptoms and family history in children and young adults with sudden cardiac arrest. J Am Board Fam Med 25(4):408. https://doi.org/10. 3122/jabfm.2012.04.110225

7. Maron BJ, Roberts WC, Epstein SE (1982) Sudden death in hypertrophic cardiomyopathy: a profile of 78 patients. Circulation. 65(7):1388. https://doi. org/10.1161/01.CIR.65.7.1388

8. Maron MS, Olivotto I, Zenovich AG et al (2006) Hypertrophic cardiomyopathy is predominantly a disease of left ventricular outflow tract obstruction. Circulation. 2008:406. https://doi.org/10.1161/CIRCULATIONAHA. 106.644682

9. Cox A, Cox AT, Folkes F, Sharma S, Boos C (2015) Screening for cardiac disease in potential recruits to the British Army screening for cardiac disease in potential recruits to the British Army. https://doi.org/10.1136/jramc-2015000532

10. La Gerche A, Baggish AL, Knuuti J et al (2013) Cardiac imaging and stress testing asymptomatic athletes to identify those at risk of sudden cardiac death. JACC Cardiovasc Imaging 6(9):993. https://doi.org/10.1016/j.jcmg. 2013.06.003

11. Rynes SL, Bretz RD, Gerhart B (1991) The importance of recruitment in job choice: a different way of looking. Pers Psychol 44(3):487. https://doi.org/10. 1111/j.1744-6570.1991.tb02402.x

12. Williams EA, Pelto HF, Toresdahl BG, Prutkin JM, Owens DS, Salerno JC, Harmon KG, Jonathan A (2019) Performance of the American Heart Association (AHA) 14-point evaluation versus electrocardiography for the cardiovascular screening of high school athletes: a prospective study. J Am Heart Assoc 8(14):e012235. https://doi.org/10.1161/JAHA.119.012235

13. Lang RM, Badano LP, Mor-Avi V et al (2015) Recommendations for cardiac chamber quantification by echocardiography in adults (2015). Eur Heart J Cardiovasc Imaging 16(3):233. https://doi.org/10.1093/ehjci/jev014

14. Lindekleiv H, Løchen M-L, Mathiesen EB et al (2013) Echocardiographic screening of the general population and long-term survival a randomized clinical study. JAMA Intern Med 173(17):1592-1598. https://doi.org/10.1001/ jamainternmed.2013.8412

15. Corrado D, Pelliccia A, Bjørnstad HH et al (2018) Cardiovascular preparticipation screening of young competitive athletes for prevention of sudden death: proposal for a common European protocol consensus statement of the study Group of Sports Cardiology of the working Group of Cardiac Rehabilitation. J Sci Med Sport 10:516-524. https://doi.org/10.1093/ eurheartj/ehi108

16. Douglas PS, Garcia MJ, Haines DE et al (2011) American College of Cardiology Foundation appropriate use criteria task force; American Society of Echocardiography; American Heart Association; American Society of Nuclear Cardiology; Heart Failure Society of America; Heart Rhythm Society; Society for Cardiovascular Angiography and Interventions; Society of Critical Care Medicine; Society of Cardiovascular Computed Tomography; Society for Cardiovascular Magnetic Resonance. ACCF/ASE/AHA/ASNC/HFSA/HRS/ SCAI/SCCM/SCCT/SCMR 2011 appropriate use criteria for echocardiography: a report of the American College of Cardiology Foundation appropriate use criteria task force, American Society of Echocardiography, American Heart Association, American Society of Nuclear Cardiology, Heart Failure Society of America, Heart Rhythm Society, Society for Cardiovascular Angiography and Interventions, Society of Critical Care Medicine, Society of Cardiovascular Computed Tomography, and Society for Cardiovascular Magnetic Resonance endorsed by the American college of chest physicians. J Am Coll Cardiol 57(9):1126-1166

\section{Publisher's Note}

Springer Nature remains neutral with regard to jurisdictional claims in published maps and institutional affiliations.

\section{Submit your manuscript to a SpringerOpen ${ }^{\circ}$ journal and benefit from:}

- Convenient online submission

- Rigorous peer review

- Open access: articles freely available online

- High visibility within the field

- Retaining the copyright to your article

Submit your next manuscript at $\boldsymbol{\nabla}$ springeropen.com 